\title{
Continuous Voltage Tunability of Intersubband Relaxation Times in Coupled SiGe Quantum Well Structures Using Ultrafast Spectroscopy
}

\author{
P. Rauter, ${ }^{1}$ T. Fromherz, ${ }^{1}$ N. Q. Vinh, ${ }^{2}$ B. N. Murdin, ${ }^{3}$ G. Mussler ${ }^{4}$ D. Grützmacher, ${ }^{4}$ and G. Bauer ${ }^{1}$ \\ ${ }^{1}$ Institute of Semiconductor and Solid State Physics, University of Linz, Linz, Austria \\ ${ }^{2}$ FOM Institute for Plasma Physics Rijnhuizen, Nieuwegein, The Netherlands \\ ${ }^{3}$ Advanced Technology Institute, University of Surrey, Guildford GU2 7XH, United Kingdom \\ ${ }^{4}$ Forschungszentrum Jülich, Jülich, Germany
}

(Received 25 September 2008; published 6 April 2009)

\begin{abstract}
We demonstrate continuous voltage control of the nonradiative transition lifetime in semiconductor heterostructures. The results were obtained by picosecond time-resolved experiments on biased SiGe valence band quantum well structures using a free electron laser. By varying the applied voltage, the intersubband hole relaxation times for quantum well structures were varied by a factor of 2 as the wave functions and their overlaps were tuned. The range of magnitudes for the lifetime indicates a possible route to silicon-based quantum cascade lasers.
\end{abstract}

PACS numbers: 78.67.De, 73.21.-b, 73.50.Pz, 78.47.-p

The choice of media with desirable electronic transition rates is critical to laser operation, and since the invention of the ruby laser physicists have sought to find atoms, molecules, and crystals with lucky combinations of energies and rates. Semiconductor heterostructures offer a means of intelligent control of lifetimes and transition energies. The carrier dynamics in such structures are central to many laser physics effects of recent topical interest such as cavity quantum electrodynamics, femtosecond pulse generation, single photon generation, etc. [1-3]. Quantum cascade lasers (QCLs) are based on intersubband transitions [4], and this type of transition provides tremendous control over the state energies and wave functions, and hence on overlaps and lifetimes. Modifying the design of the structure from spatially direct to indirect (diagonal) radiative transitions in QCL structures provides a means of increasing the lifetime of the excited state over many orders of magnitude, in order to achieve population inversion and lasing [5-7]. Although control via structural design is attractive, voltage control of the lifetime within a single structure offers a way to perform novel dynamics experiments and has potential benefits for understanding incoherent laser dynamics.

Work on the precise determination of relaxation times in the SiGe system has been stimulated by recent picosecond pulsed measurements on passive $\mathrm{SiGe}$ intersubband structures suggesting that light-hole-heavy-hole (LH-HH) transitions might be promising for realizing a SiGe QCL [8,9], which has yet to be demonstrated despite reported intersubband electroluminescence [10]. QCL devices based on diagonal transitions exhibit a strong dependence of both emitted wavelength and output power on the applied bias, which supports the idea of voltage control of transition rates, but the results must be deconvoluted from the effects of varying the injection and extraction rates, wavelength dependent cavity loss, etc. [11]. Ultrafast relaxation times of electrically biased structures have become experimentally accessible by recent photocurrent (PC) pump-pump experiments [12].

In this work we report time-resolved relaxation of the excited state population in a quantum well (QW) structure. The experimentally observed decay time is voltage tuned as the relaxing transition is changed from a spatially direct
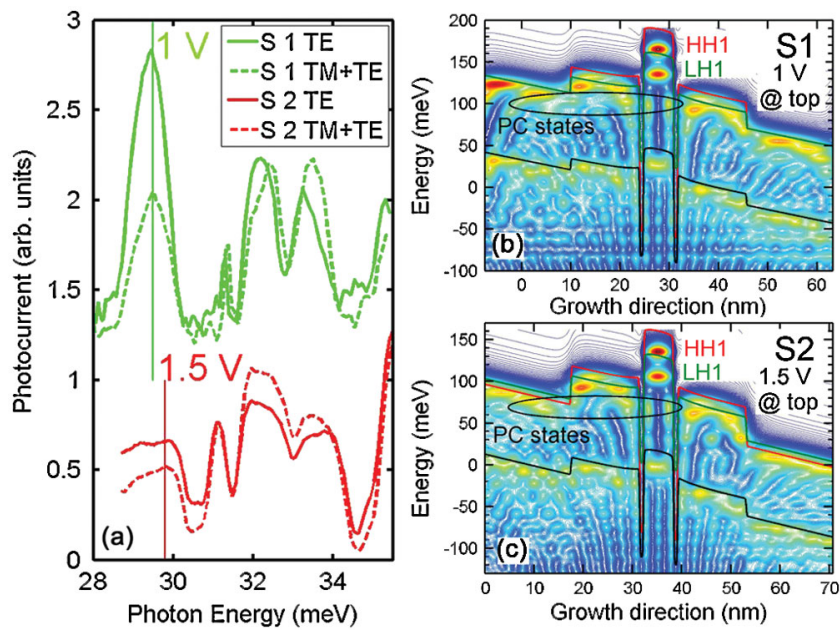

FIG. 1 (color). Two-photon PC experiments and simulation results. In (a), two-photon PC spectra obtained by varying the FEL wavelength in different polarizations are presented. The spectra for $S 1$ and $S 2$ are offset vertically for clarity. (b),(c) show the calculated $\mathrm{HH}, \mathrm{LH}$, and split-off (red, green, black) band edges at $1 \mathrm{~V}(S 1)$ and $1.5 \mathrm{~V}(S 2)$ bias and a contour plot of the absolute squared wave functions at their eigenenergy values for samples $S 1$ and $S 2$, respectively. The calculated LH1-HH1 energy differences are consistent with the spectral peak positions. The photoconducting final states of the two-photon absorption are highlighted by ellipses. The vertical lines in (a) indicate the energy at which the time-resolved measurements were performed. 
to an indirect one, and thus we directly monitor the lifetime-increasing concept of diagonal transitions. The measurements were performed using far-infrared, picosecond pulses from the free electron laser (FEL) "FELIX" at FOM Rijnhuizen $[12,13]$.

The SiGe QW samples were grown pseudomorphically by molecular beam epitaxy on a $\mathrm{Si}_{0.8} \mathrm{Ge}_{0.2}$ (100) pseudosubstrate. One period of each sample structure consists of a deep central QW separated symmetrically by Si barriers from two shallow side wells. The active region of sample $S 1$ consists of a $61 \AA$ wide $\mathrm{Si}_{0.69} \mathrm{Ge}_{0.31}$ central well separated by $8 \AA$ thick barriers from $140 \AA$ wide $\mathrm{Si}_{0.76} \mathrm{Ge}_{0.24}$ side wells. Adjacent active regions are separated by $200 \AA$ wide $\mathrm{Si}_{0.8} \mathrm{Ge}_{0.2}$ spacers. The structure of sample $S 2$ differs from that of $S 1$ only in the spacer between active regions, which has a width of $350 \AA$ and consists of $\mathrm{Si}_{0.82} \mathrm{Ge}_{0.18}$ for $S 2$. For both samples the central wells and adjacent $\mathrm{Si}$ barriers were boron doped with a concentration of $10^{18} \mathrm{~cm}^{-3}$. Each sample consists of 15 periods of the described structures sandwiched between $\mathrm{Si}_{0.8} \mathrm{Ge}_{0.2}$ contact layers of a doping concentration of $4 \times 10^{18} \mathrm{~cm}^{-3}$. For vertical PC measurements the samples were processed into mesas $\left(S 1,400 \times 400 \mu \mathrm{m}^{2} ; S 2,900 \times 900 \mu \mathrm{m}^{2}\right)$ and contacted by $\mathrm{Al}: \mathrm{Si}$ metallization. The active structures were illuminated through polished side facets $\left[30^{\circ}\right.$ with respect to the (001) surface] by either completely TE polarized radiation or by radiation with a TE-TM intensity ratio of 1:3. In Figs. 1(b) and 1(c), the band structures of the samples are shown for the $\mathrm{HH}, \mathrm{LH}$, and split-off band edges. They were calculated using a six band k.p envelope function model for the valence bands, including strain according to Ref. [14]. The absolute square of the wave functions is shown as a contour plot, where the spreading along the energy axis indicates the energy broadening of the state. The HH1-LH1 transition energies of the central wells were calculated to be around $30 \mathrm{meV}$ for both samples. At low temperatures and in the absence of radiation, holes are confined in the central well's HH1 states. Resonant FEL radiation excites charge carriers into LH1, a process which is only strong in TE polarization. At low voltages the LH1 state is strongly confined to the central well. In order to contribute to a PC, charge carriers excited to LH1 have to absorb a second photon into energetically higher states, that are only weakly confined in a biased structure. The samples were designed in such a way as to make the generation of PC by a two-photon process of energetically equal photons possible. The weakly confined final states are built up by mixed $\mathrm{HH} 2$ and $\mathrm{LH} 2$ states; consequently, the transition from the confined LH1 state into delocalized states is strong in both polarizations. Figure 1(a) shows normalized PC spectra based on multiphoton absorption at a sample temperature of $4.2 \mathrm{~K} \mathrm{ob-}$ tained by sweeping the FEL wavelength. The FEL radiation was coupled into the sample via the $30^{\circ}$ facet; the photocurrent spectra for completely TE polarized radiation are shown as solid lines, those for TM + TE as broken lines. For $S 1$ (green lines) the measured spectra show a clear resonance in TE polarization at the calculated energy, whereas for the structurally nearly identical $S 2$ (red line) the PC signal at the calculated resonance is significantly weaker. This is due to the energetically higher and spatially wider barriers formed by the spacer layer between well regions of $S 2$ in comparison to $S 1$. The states excited by two-photon absorption are more strongly confined for $S 2$ than for $S 1$, as can be concluded from the more pronounced spatial carrier density modulation of $S 2$ in Fig. 1(c) highlighted by the ellipse, and therefore contribute less to the PC at similar bias $(S 1,12 \mathrm{kV} / \mathrm{cm} ; S 2$, $14 \mathrm{kV} / \mathrm{cm})$. This demonstrates the design trade off between the required confinement of the LH ground states of the side wells (allowing a spatial shift of the LH1 state of the central well without causing a direct photocurrent) and the detrimental confinement of their excited states (required as final states for the generation of PC). For a fixed side QW width, the crucial parameter for the optimization of these two contrary requirements is the energy barrier at the interface between spacer and side QW.

PC pump-pump experiments were performed in order to determine the LH1-HH1 relaxation time, which has been reported in [15] to be mainly determined by alloy scattering with an expected relaxation time about $10 \mathrm{ps}$ for energy level differences below the optical phonon energy ( $\mathrm{Si}-\mathrm{Si}$, $58 \mathrm{meV}$; Ge-Ge, $36 \mathrm{meV}$ ). FEL micropulses with a FWHM of about $10 \mathrm{ps}$ and a spot diameter of $1.4 \mathrm{~mm}$ were employed. The FEL beam was tuned to the respective HH1LH1 resonance energy of the samples $(S 1,29.5 \mathrm{meV}$;

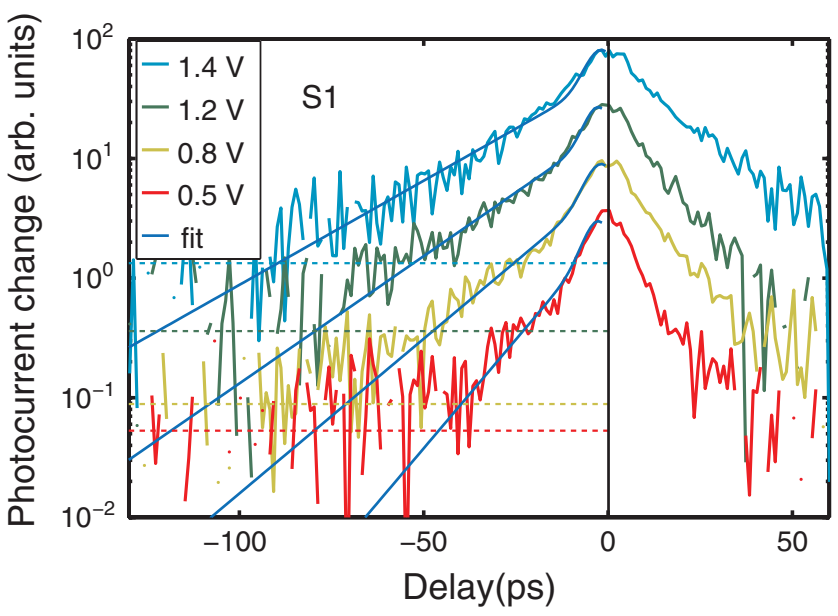

FIG. 2 (color). Logarithmic plot of the PC through sample $S 1$ as a function of the delay between pulse $1(\mathrm{TM}+\mathrm{TE})$ and pulse 2 (TE) for different bias voltages at a temperature of $4 \mathrm{~K}$ (positive delay means pulse 1 arrives second). A constant background equivalent to the current integral over the two noninteracting pulses (i.e., delays $>100 \mathrm{ps}$ ) is subtracted separately for positive and negative delays and the traces are offset vertically for clarity. The additional, nonlinear PC signal clearly decays slower for increasing bias. The dotted lines indicate the measurements' noise levels. The FEL micropulse energy density was $5 \mu \mathrm{J} / \mathrm{cm}^{2}$ for both pulses. Also shown are results of the fitting procedure described in the text (blue lines). 
$S 2,29.8 \mathrm{meV}$ ). It entered the optical setup in TE polarization and was split by a beam splitter. The polarization of beam 1 was turned by $90^{\circ}$. Beam 2 remained in purely TE polarization and was reflected by a movable mirror allowing adjustment of the delay between TE and TM $+\mathrm{TE}$ pulse with subpicosecond resolution. Before being coupled into the sample waveguide, the beams were made collinear again using a second beam splitter, where the setup was balanced in order to achieve equivalent intensity of both beams at the sample position. The integrated PC through the variably biased sample originating from the combined effects of the delayed TE and TM + TE micropulses was measured as a function of the delay. The cross-polarization of the FEL beams broke the symmetry of the experiment with respect to the sign of the delay.

For $S 1$ the results of the pump-pump measurements after subtracting backgrounds that correspond to the PC signal for large delays are presented in Fig. 2 for a series of voltages applied to the sample contacts. Because of the presence of heating effects on a time scale much larger than the maximum delay time of the experiment, different backgrounds for positive and negative delays had to be subtracted. The curves show an asymmetry with respect to the sign of the delay, which is due to the polarization dependence of the transition involved. The curves in Fig. 2 clearly exhibit a systematic dependence of the PC decay behavior on the applied bias. The PC signal decays significantly more slowly with increasing voltage. The response model used to extract the quantitative PC decay times from the pump-pump data is presented in Eqs. (1)(3). The nonlinear photocurrent for a given delay $\Delta t$ in Eq. (1) is obtained by integrating over two current contributions: first, the current generated by excitation from the nonequilibrium LH state occupation $n_{\text {occ }}(t)$ established by the first pulse; and second, the current generated by instantaneous, coherent two-photon absorption accounted for by $I_{\text {pulse }}\left(t^{\prime}\right) I_{\text {pulse }}\left(t^{\prime}+\Delta t\right)$. $I_{\text {conv }}(t)$, which is proportional to $n_{\text {occ }}(t)$, is gained by convoluting $I_{\text {pulse }}(t)$ with a decay function, where $\tau_{\text {decay }}$ is the nonequilibrium occupation's relaxation time. In our model, the temporal width of the Gaussian FEL pulse $\tau_{\text {pulse }}$ and the ratio between the PC contributions of the instantaneous two-photon absorption and the occupation of intermediate states $\frac{p_{2}}{p_{1}}$ have been assumed bias independent (global parameters). The relaxation time $\tau_{\text {decay }}$ and the PC background $j_{0}$ are allowed to vary with bias voltage, where the confidence region of $j_{0}$ constitutes less than $1 \%$ of its fit value. The global parameters are chosen by minimizing the average variances of the least square curve fits for the various voltages.

$j(\Delta t)=\int_{-\infty}^{\infty} I_{\text {pulse }}\left(t^{\prime}+\Delta t\right)\left[p_{1} I_{\text {conv }}\left(t^{\prime}\right)+p_{2} I_{\text {pulse }}\left(t^{\prime}\right)\right] d t^{\prime}+j_{0}$

$$
I_{\text {conv }}(t)=\int_{-\infty}^{t} I_{\text {pulse }}\left(t^{\prime}\right) e^{-\left(\left|t-t^{\prime}\right| / \tau_{\text {deay }}\right)} d t^{\prime} \sim n_{\text {occ }}(t)
$$

$$
I_{\text {pulse }}(t)=e^{-\left(t^{2} / \tau_{\text {pulse }}^{2}\right)} .
$$

Figure 3 shows the variation of the PC decay time $\tau_{\text {decay }}$ with the bias for both samples. The decay times are obtained from a series of pump-pump experiments, a selection of which is shown for $S 1$ in Fig. 2, by fitting the curves for negative delay according to the model described above. For $S 1$ the decay time rises continuously from 12 to $25 \mathrm{ps}$ when changing the applied voltage (field) from $0.2 \mathrm{~V}$ $(2.4 \mathrm{kV} / \mathrm{cm})$ to $1.4 \mathrm{~V}(16.8 \mathrm{kV} / \mathrm{cm})$. The results show a voltage-induced change of the decay time of a PC signal by a factor of 2 induced by the first (TE) FEL pump pulse. The error bars confirm the significance of the observed decay time rise. The indicated confidence region was calculated using the Jacobian and the deviation of the fit from the measurement curve assuming normal distribution. As expected from the structural similarities between $S 1$ and $S 2$ and seen in Fig. 3, their PC relaxation changes with the applied voltage in a similar way. By tuning the voltage (field) applied to $S 2$ from $0.5 \mathrm{~V}(4.7 \mathrm{kV} / \mathrm{cm})$ to $2 \mathrm{~V}$ $(18.8 \mathrm{kV} / \mathrm{cm})$, the relaxation time changes continuously by a factor of 2 .

The origin of the voltage tunability of the $\mathrm{PC}$ relaxation time can be found in the samples' band structure, which is shown for $S 1$ in Fig. 4. On a time scale exceeding that of the FEL pulse duration in Fig. 2, the nonlinear PC originates from a nonequilibrium occupation of excited states established by the first pump pulse. For negative delays in Fig. 2, the purely TE polarized pulse is the first one to interact with the sample. In this polarization, the HH1-LH1 transition is allowed, and thus a nonequilibrium population

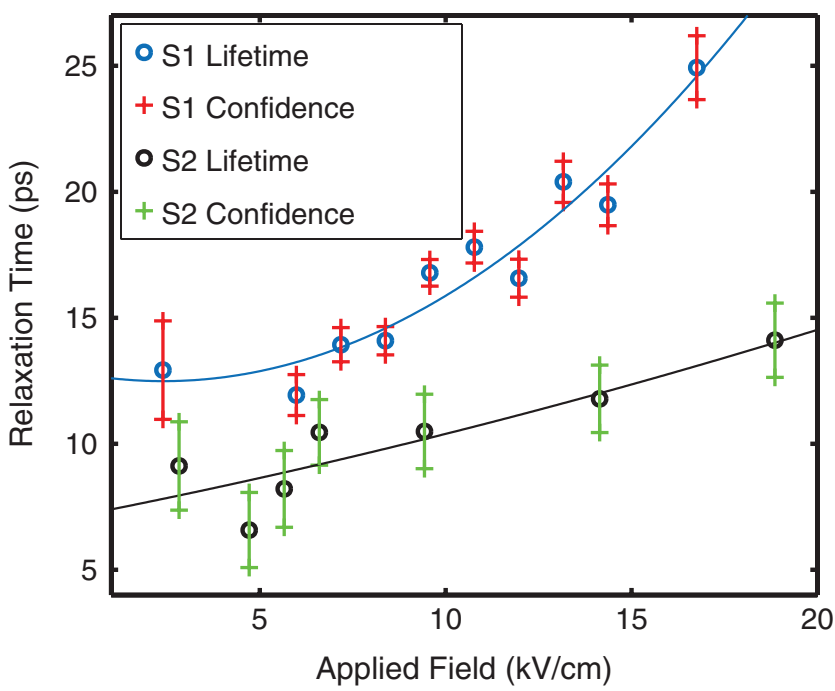

FIG. 3 (color). Voltage dependence of the LH1-HH1 relaxation time. The dots show a clear, bias induced monotonic rise by a factor of 2 of both samples' intersubband relaxation times extracted from the pump-pump data gained at $4 \mathrm{~K}$. The red error bars and the green error bars indicate the $68 \%$ confidence limits of the fitted relaxation times. The solid lines serve as a guide for the eye. 

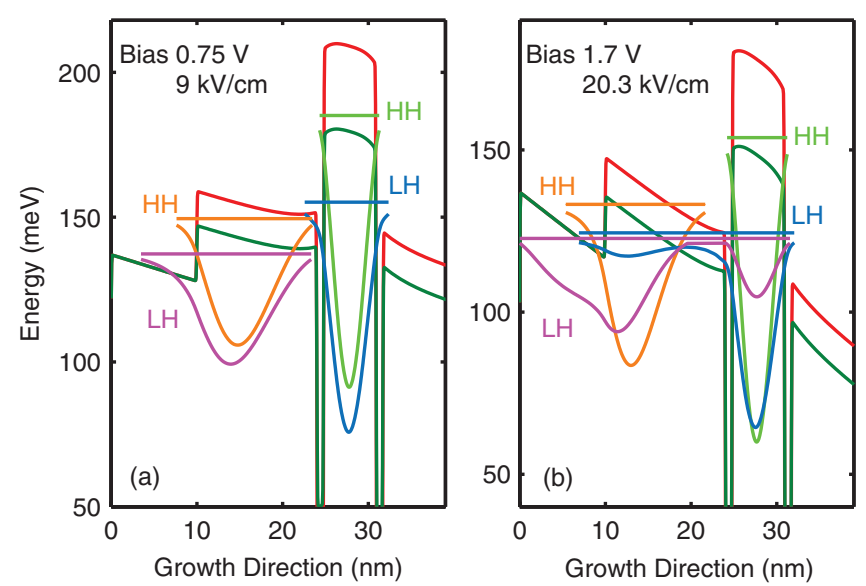

FIG. 4 (color). Change in the band structure of $S 1$ for applied voltages of 0.75 and $1.7 \mathrm{~V}$. The plots show the calculated heavy and light (red lines and green lines) hole band edges and the absolute squared wave functions at their eigenenergy values. In (a) the LH1 state of the central well is confined, while it couples to the LH state of the side well in (b). Therefore, for a bias of $1.6 \mathrm{~V}$ the center of mass for holes excited from $\mathrm{HH} 1$ into LH states is shifted from the central well, the relaxing transition to the $\mathrm{HH}$ ground state is a diagonal one with a reduced hole density overlap. Consequently, the relaxation time increases from (a) to (b).

of the LH1 state of the central well is established. This nonequilibrium occupation decays exponentially with the LH1-HH1 relaxation time. From the LH1 state, holes can be excited to the continuum in both TE and TM polarization. If the second, $\mathrm{TE}+\mathrm{TM}$, pulse hits the structure before the LH1 carriers have relaxed, the integrated PC increases proportionally to the number of residual holes in the LH1 state. Consequently, the measured integrated PC decays exponentially with the delay between the two pump pulses. Since the LH1-HH1 selection rules only weakly allow a LH1 population generated by a TM pulse, the contribution of the LH1-occupation to the PC signal is much weaker if the TM + TE pulse arrives first (positive delays in Fig. 2) and an asymmetry with respect to the pulse order results, as observed in the experiment. However, as long as the voltage applied to the sample is low, the LH1 state is confined to the central well, as seen in Fig. 4(a). As the bias increases, the LH1 state of the central well starts coupling to the LH state of the shallow side well, shown in Fig. 4(b). Thus the occupation of excited states that are spatially shifted from the central well is built up more and more. According to calculations of the interband alloy scattering rate [16] for sample $S 1$, the overlap between the initial and final states of the scattering transition as well as the scattering rate are decreased by a factor of approximately 2 by changing the bias from $0.75 \mathrm{~V}$ [Fig. 4(a)] to $1.7 \mathrm{~V}$ [Fig. 4(b)]. In the calculation, a complete phase randomization between the excited LH state doublet established by the ultrafast ( $\ll 250 \mathrm{fs}$ ) interface roughness intraband scattering was assumed [17]. As the second FEL pulse directly monitors the LH occupation, this increase in relaxation time is directly reflected in the increase of the additional PC's decay time.

In conclusion, we report a directly observed bias tuning of intersubband relaxation times in QW structures by closely correlating the changing relaxation time of a PC signal in a FEL pump-pump experiment with the electric field-driven variation of the band structure. By continuously changing a spatially direct transition into indirect, we were able to increase the intersubband relaxation times in two SiGe QW samples of different designs. A simple model allowed the extraction of quantitative relaxation time values and the demonstration of a clear monotonic rise of these values with increasing voltage. Our experiments allowed the direct study of the influence of the wave function overlap on relaxation times within one and the same structure. In $\mathrm{SiGe} \mathrm{QWs}$, we observe a voltageinduced increase in excited state lifetimes by a factor of 2, supporting the concept of diagonal transition as a means to achieve population inversion in SiGe quantum cascade structures. In addition, our work demonstrates the necessity for time-resolved experiments on biased QW structures, as relaxation times measured on unbiased structures can differ substantially from those relevant for device operation.

We gratefully acknowledge the support by the FWF (SFB IROn F2512-N08) and GME, both Vienna, and by the Stichting voor Fundamenteel Onderzoek der Materie (FOM) in providing the required beam time on FELIX and highly appreciate the skillful assistance by the FELIX staff. This work was supported by the European Community Research Infrastructure Action under the FP6 "Structuring the European Research Area" Programme through the Integrated Infrastructure Initiative "Integrating Activity on Synchrotron and Free Electron Laser Science."

[1] J. Kröll et al., Nature (London) 449, 698 (2007).

[2] L. Sapienza et al., Phys. Rev. Lett. 100, 136806 (2008).

[3] Hyunyong Choi et al., Phys. Rev. Lett. 100, 167401 (2008).

[4] For reviews, see, e.g., C. Gmachl et al., Rep. Prog. Phys. 64, 1533 (2001); Special issue on QCL [IEEE J. Quantum Electron. 38, 509 (2002)].

[5] J. Faist et al., Nature (London) 387, 777 (1997).

[6] I. Bormann et al., Physica (Amsterdam) 21E, 779 (2004).

[7] S. Tsujino et al., IEEE J. Quantum Electron. 12, 1642 (2006).

[8] C. Pidgeon et al., Semicond. Sci. Technol. 20, L50 (2005).

[9] R. Bates et al., Appl. Phys. Lett. 83, 4092 (2003).

[10] G. Dehlinger et al., Science 290, 2277 (2000).

[11] I. Bormann et al., Appl. Phys. Lett. 83, 5371 (2003).

[12] P. Rauter et al., New J. Phys. 9, 128 (2007).

[13] P. Rauter et al., Appl. Phys. Lett. 89, 211111 (2006).

[14] T. Fromherz et al., Phys. Rev. B 50, 15073 (1994).

[15] P. Murzyn et al., Appl. Phys. Lett. 80, 1456 (2002).

[16] Z. Ikonic, P. Harrison, and R. W. Kelsall, Phys. Rev. B 64, 245311 (2001).

[17] S. Tsujino et al., Appl. Phys. Lett. 86, 062113 (2005). 